A C T A C H E M I A S C A N D I N A I CA $20 \quad(1966) \quad 197-205$

\title{
Quantitative Determination of Volatile Compounds from Food and Evaluation of the Gas Flushing Method for their Concentration
}

\author{
Ö. WAHLROOS* and O. E. NIKKILÄ \\ State Institute for Technical Research, Laboratory for Food Research and Technology, \\ Otaniemi, Finland
}

\begin{abstract}
Theoretical considerations have been applied to the head-space method and to the method of concentrating volatiles from dilute solution by flushing the sample with an inert gas and condensing the vapours from the gas stream before gas chromatography. These analytical methods are shown to become quantitative if two successive chromatograms are made from each sample, which is flushed with known volumes of gas. Simple equations were derived for calculation of the results. An equation has been deduced to describe the concentration curve in a condensation vessel containing solvent, when condensation is incomplete. The applications of the methods are discussed.
\end{abstract}

$\mathbf{G}$ as chromatographic head space analysis has been used for the determination of the concentration of volatile compounds in the gas phase above their solution.,2 In the numerous cases in which the sensitivity of gas chromatographic detectors is not sufficient for head space analysis, a frequently employed method for concentration is to pass a stream of inert gas through the sample and condense the vapours in a refrigerated tube, which may contain some solvent (e.g. a short chromatographic column or adsorbent). ${ }^{3}$ This system has also been used for preparative work.

If quantitative results are to be obtained by these methods, however, the following questions must first be answered:

Head space analysis: what are the concentrations of the compounds in the sample itself? Concentration method: what does the chromatogram obtained from the concentrate signify? Can quantitative conclusions be drawn? What are the optimum conditions for preparative concentration? The questions may be elucidated by some theoretical considerations.

* Present address: Department of Nutritional Chemistry, Helsinki University, Helsinki, Finland.

Acta Chem. Scand. 20 (1966) No. 1 


\section{THEORETICAL}

S y m b o l s

$V_{\mathrm{g} 1} \quad=$ volume of the gas phase above the sample.

$V_{\mathrm{L} 1}^{\mathrm{g} 1} \quad=$ volume of the sample. The sample may here be regarded as a liquid or solid solvent, or a mixture of these. $V_{L_{1}}$ may also represent the active surface of an adsorbent

$V_{\mathrm{g} 2}, V_{\mathrm{L}_{2}}=$ corresponding volumes in the condensation vessel

$C_{\mathrm{g1}}^{\mathrm{g2}} \quad=$ concentration of the compound to be analysed in the gas phase above the sample

$C_{\mathrm{g2}} \quad=$ gas phase concentration of the compound in the concentration vessel

$C_{\mathrm{go}} \quad=$ starting concentration of the compound above the sample $C_{\mathrm{L}, 0}^{\mathrm{g0}}, C_{\mathrm{L}, 1}, C_{\mathrm{L}, 2}=$ corresponding concentrations in the liquid phases (or solid, or adsorbent)

$v \quad=$ volume of gas led through the sample

$k_{1}, k_{2} \quad=$ distribution coefficients of the compound in the sample and the condensation vessel. $k_{1}$ is to be regarded as an overall coefficient between the sample and its gas phase when the sample is of heterogeneous material. The $k$ 's are $V_{1}, V_{2} \quad \begin{aligned} & \text { regarded as linear over the concentration range in question } \\ & \text { effective volumes of sample and condensation vessels, }\end{aligned}$

$$
V_{1}=V_{\mathrm{g} 1}+k_{1} \cdot V_{\mathrm{L}, 1}
$$

$W \quad=$ total amount of a compound in the sample

\section{Head space analy is}

As mentioned, a head space chromatogram gives information only about the concentrations of the components in the gas phase above the sample. To know the concentrations in the sample itself, we should determine the distribution coefficient of each compound to be determined from the chromatogram. This should be done for each sample, because the solvent properties of the sample may vary (e.g., the fat content of some foods varies within wide limits). The determination of the distribution coefficients need not, however, be very laborious. It is possible to calculate the coefficients for all components visible on the chromatogram from two successive runs. It has previously been shown 4 that the concentration of a volatile compound decreases logarithmically when a gas is led through the sample at such a rate that equilibrium is attained between sample and gas, i.e.

$$
\ln C_{\mathrm{g} 1}=\ln C_{\mathrm{g} 0}-\left(v / V_{1}\right)
$$

To give a true picture of the concentrations in the gas phase, the volume of the head space sample withdrawn must be small in comparison with the effective volume of the sample, as may be deduced from eqn. 4 . If now, after making a head space chromatogram, and blowing a volume $v$ of carrier gas through the sample, a second head space chromatogram is made, we have from (1) 


$$
C_{\mathrm{g} 0} V_{1}=\frac{C_{\mathrm{g} 0} v}{\ln C_{\mathrm{g} 0}-\ln C_{\mathrm{g} 1}}=W
$$

$C_{\mathrm{g} 0}$ and $C_{\mathrm{g} 1}$ are obtained from the areas of the corresponding peaks in the first and the second chromatogram, i.e., areas I and II in Fig. 1, when the specific sensitivity of the detector and the injected volume are taken into account. (2) is the total amount in the sample of the compound under investigation.

If the first chromatogram is made when a volume $v_{\mathrm{a}}$, and the second when a volume $v_{\mathrm{b}}$ of carrier gas has passed through the sample (Fig. 1), the amount of the compound originally present in the sample is obtained by writing (1) for $C_{\mathrm{ga}}, v_{\mathrm{a}}$ and $C_{\mathrm{gb}}, v_{\mathrm{b}}$, combining and rearranging:

$$
W=C_{\mathrm{ga}} \cdot \frac{v_{\mathrm{b}}-v_{\mathrm{a}}}{\ln \left(C_{\mathrm{ga}} / C_{\mathrm{gb}}\right)} \cdot\left(C_{\mathrm{ga}} / C_{\mathrm{gb}}\right) \exp \left[v_{\mathrm{a}} /\left(v_{\mathrm{b}}-v_{\mathrm{a}}\right)\right]
$$

Quantitative determination by the concentration method when the eluted vapours aretotally condensed

It may be asked, why not elute the volatiles totally and make one chromatogram? However, the distribution coefficients very often have values from 100 to 10000 . In these cases, excessive volumes of carrier gas have to be used, total trapping will be difficult to achieve, and with aqueous samples, large volumes of water will be condensed, disturbing the gas chromatography.

It is easier to elute a part of the volatiles sufficiently large to render the compounds visible on the chromatogram. Then it is necessary to make two chromatograms to determine the original concentrations of the compounds in the gas phase above the sample, and the distribution coefficients (these would not be obtained on total elution).
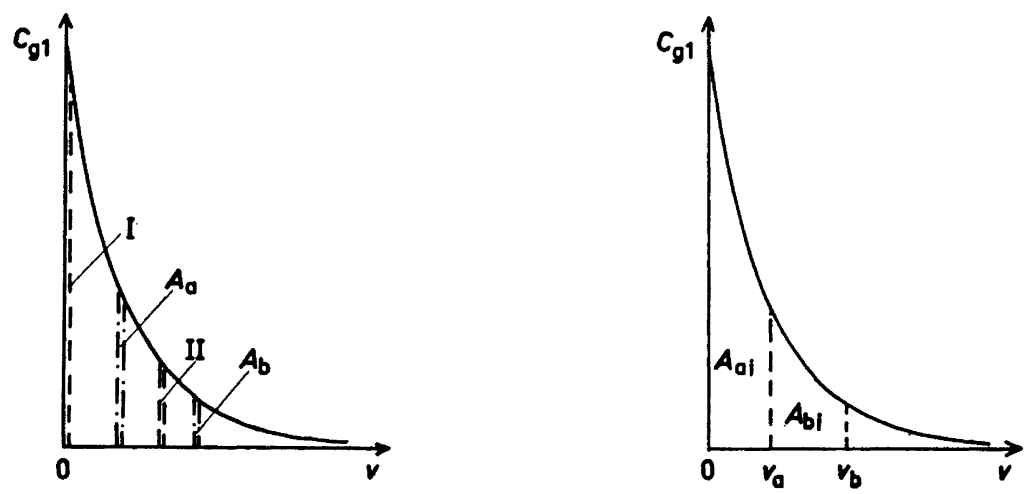

Figs. 1. and 2. Decrease of vapour concentration above sample flushed with carrier gas. Areas corresponding to peak areas on chromatography of limited volumes.

Acta Chem. Scand. 20 (1966) No. 1 
From Fig. 2 it is seen that the peak area on the first chromatogram of an eluted compound $\mathrm{i}$ will correspond to the area $A_{\text {ai }}$, and the peak area of the same compound on the second chromatogram to $A_{\mathrm{bi}}$, when the volatiles are trapped from successive volumes $v_{\mathrm{a}}$ and $v_{\mathrm{b}}-v_{\mathrm{a}}$ of carrier gas.

$$
\begin{gathered}
A_{\mathrm{ai}}=\int_{0}^{v_{\mathrm{a}}} C_{\mathrm{g} 1 \mathrm{i}} \mathrm{d} v=\int_{0}^{v_{\mathrm{a}}} C_{\mathrm{g} 0 \mathrm{i}} \cdot\left[\exp \left(-v / V_{1 \mathrm{i}}\right)\right] \mathrm{d} v= \\
V_{1 \mathrm{i}} C_{\mathrm{g} 0 \mathrm{i}}\left[1-\exp \left(-v_{\mathrm{a}} / V_{1 \mathrm{i}}\right)\right]
\end{gathered}
$$

Writing the corresponding expression for $A_{\mathrm{bi}}$, we have for the ratio between the peak areas on the two chromatograms

$$
A_{\mathrm{ai}} / A_{\mathrm{bi}}=\frac{1-\exp \left(-v_{\mathrm{a}} / V_{1 \mathrm{i}}\right)}{\exp \left(-v_{\mathrm{a}} / V_{1 \mathrm{i}}\right)-\exp \left(-v_{\mathrm{b}} / V_{1 \mathrm{i}}\right)}
$$

If the successive eluting volumes are equal, $v_{\mathrm{b}}=2 v_{\mathrm{a}}$, we have from (5)

$$
V_{1 \mathrm{i}}=v_{\mathrm{a}} /\left[2.3\left(\log A_{\mathrm{ai}}-\log A_{\mathrm{bi}}\right)\right]
$$

From the chromatographic peak areas and the gas volumes used for elution we can thus calculate the effective volume of the sample for compound $i$, and the distribution coefficient if the gas and liquid (or solid) volumes of the sample are known.

If we combine eqns. (4) and (6), we obtain the simple formula

$$
W=V_{1 \mathrm{i}} C_{\mathrm{g} 0 \mathrm{i}}=\frac{A_{\mathrm{ai}}{ }^{2}}{A_{\mathrm{ai}}-A_{\mathrm{bi}}} K^{\prime}
$$

for the total amount of compound i in the sample. $K^{\prime}$ is the specific sensitivity of the detector as weight of compound per unit area under the peak. The only requirement is that the sample should be eluted with two equal volumes of carrier gas. In practical work, however, the effect of an error in the measurement of the peak areas on the chromatograms must be allowed for. For example, if the effective volume for the compound in question is 10000 and the eluting volumes are $500 \mathrm{ml}$, a $1 \%$ error in peak area measurement may lead to an error of about $100 \%$ in $W$, while this error is only $1.6 \%$ when the eluting volumes are $5000 \mathrm{ml}$. The larger the effective volume, the smaller is the slope of the curve (Fig. 2), and the larger the eluting volumes have to be. The same applies to the head space method described in the previous section.

What conclusions can be drawn from analyses by the concentration column method when only one chromatogram is made? From (4) it is seen that the peak area of a compound $i$ is proportional to the concentration of $i$ in the samples, provided that the samples are always eluted with the same gas volume at the same temperature, the sample and the head space volumes are always the same, and the solvent properties of the samples (for $i)$ are constant. Thus comparisons between the relative concentrations of $i$ in different samples might be made. The other conditions are easily standardized, but the solvent properties of the samples are often variable. For the peak area ratio of two different compounds $\mathrm{i}$ and $\mathrm{p}$, we have from (4)

$$
A_{\mathrm{ai}} / A_{\mathrm{ap}}=C_{\mathrm{g} 0 \mathrm{i}} V_{1 \mathrm{i}}\left[1-\exp \left(-v / V_{1 \mathrm{i}}\right)\right] / C_{\mathrm{g} 0 \mathrm{p}} V_{1 \mathrm{p}}\left[1-\exp \left(-v / V_{1 \mathrm{p}}\right)\right]
$$


For limited values of $v$ it is thus not possible to compare the concentrations of $i$ and $p$ accurately from one chromatogram. As $v / V_{1} \rightarrow 0$, we approach the conditions for the head-space chromatogram. As $v / V_{1} \rightarrow \infty$, total elution of the compounds is approached.

A condition for the use of these quantitative methods is, of course, that the compound to be determined is separated from other compounds on the chromatogram. It is seen from (5) that if several successive elutions are made and the condensates chromatographed, $A_{\mathrm{ai}} / A_{\mathrm{bi}}, A_{\mathrm{bi}} / A_{\mathrm{ci}}$; etc., will be constant if $i$ appears alone or if it is superimposed on another compound that has the same distribution coefficient in the sample, too. If the chromatographic peak of $i$ is superimposed on a compound that has a different partition coefficient in the sample, the peak area ratios will drift towards a constant value $A_{\mathrm{ni}}$ / $A_{(\mathrm{n}+1) \mathrm{i}}$ if several elutions are made with equal volumes. The original concentration of the compound with the highest $k$ in the sample may then be calculated from

$$
\begin{gathered}
V_{1}=v_{n+1}-v_{\mathrm{n}} /\left(\log A_{\mathrm{n}}-\log A_{\mathrm{n}+1}\right) \\
A_{\mathrm{n}}=V_{1} C_{\mathrm{g} 0}\left[\exp \left(-v_{\mathrm{n}} / V_{1}\right)-\exp \left(-v_{\mathrm{n}-1} / V_{1}\right)\right]
\end{gathered}
$$

where $v_{n-1}$ is the total eluting volume until the constant ratio is achieved, and $v_{n}$ and $v_{n+1}$ the two following total elution volumes. The other superimposed compound may be found by subtraction.

Concentration of volatilesbythecollectioncolumn method with incomplete condensation from the carrier gas stream

In collection the aim is to keep the effective volume $V_{2}$ of the condensation tube higher than the effective volume $V_{1}$ of the sample. Naturally, the concentration of a volatile compound in the collection column will pass through a maximum when a continuous stream of carrier gas is led through the sample and collection tubes. The exact form of this concentration vs. gas volume curve will be deduced.

$C_{\mathrm{g} 2}$ is a function of the concentration $C_{\mathrm{g} 1}$ in the gas stream arriving at the collection tube and the volume $v$ of carrier gas which has passed. The rate of change of $C_{\mathrm{g2}}$ with regard to $v$ is now given by the total differential

$$
\frac{\mathrm{d} C_{\mathbf{g} 2}}{\mathrm{~d} v}=\frac{\partial C_{\mathrm{g} 2}}{\partial v}+\frac{\partial C_{\mathrm{g} 2}}{\partial C_{\mathrm{g} 1}} \times \frac{\mathrm{d} C_{\mathrm{g} 1}}{\mathrm{~d} v}
$$

The amount of material brought by the gas stream to the collection tube equals the increase of material in the collection tube and the amount flushed away from it by the gas:

or

$$
C_{\mathrm{g} 1} \mathrm{~d} v=V_{\mathrm{g} 2} \mathrm{~d} C_{\mathrm{g} 2}+V_{\mathrm{L}, 2} \mathrm{~d} C_{\mathrm{L}_{2}}+C_{\mathrm{g} 2} \mathrm{~d} v
$$

i.e.

$$
\left(C_{\mathrm{g} 1}-C_{\mathrm{g} 2}\right) \mathrm{d} v=V_{2} \mathrm{~d} C_{\mathrm{g} 2}
$$

$$
\frac{\partial C_{\mathrm{g} 2}}{\partial v}=\frac{C_{\mathrm{g} 1}-C_{\mathrm{g} 2}}{V_{2}}
$$

Acta Chem. Scand. 20 (1966) No. 1 
When $v$ is constant, no concentration changes occur in the sample and collection vessels, $\partial C_{\mathbf{g 2}} / \partial C_{\mathbf{g} 1}=0$, and

$$
\mathrm{d} C_{\mathrm{g} 2}=\frac{C_{\mathrm{g} 1}-C_{\mathrm{g} 2}}{V_{2}} \mathrm{~d} v
$$

Writing eqn. 1 in the exponential form $C_{\mathrm{g} 1}=C_{\mathrm{g} 0}\left[\exp \left(-v / V_{1}\right),\right]$ and substituting in (12), we obtain

$$
\frac{\mathrm{d} C_{\mathrm{g} 2}}{\mathrm{~d} v}+\frac{1}{V_{2}} \cdot C_{\mathrm{g} 2}=\frac{1}{V_{2}} \cdot C_{\mathrm{g} 0}\left[\exp \left(-v / V_{1}\right)\right]
$$

The solution of this linear differential equation, when $C_{\mathrm{g} 2}=0$ for $v=0$ is

$$
C_{\mathrm{g} 2}=\frac{C_{\mathrm{g} 0} V_{1}}{V_{1}-V_{2}} \cdot\left[\exp \left(-v / V_{1}\right)-\exp \left(-v / V_{2}\right)\right]
$$

This is graphically represented in Fig. 3.

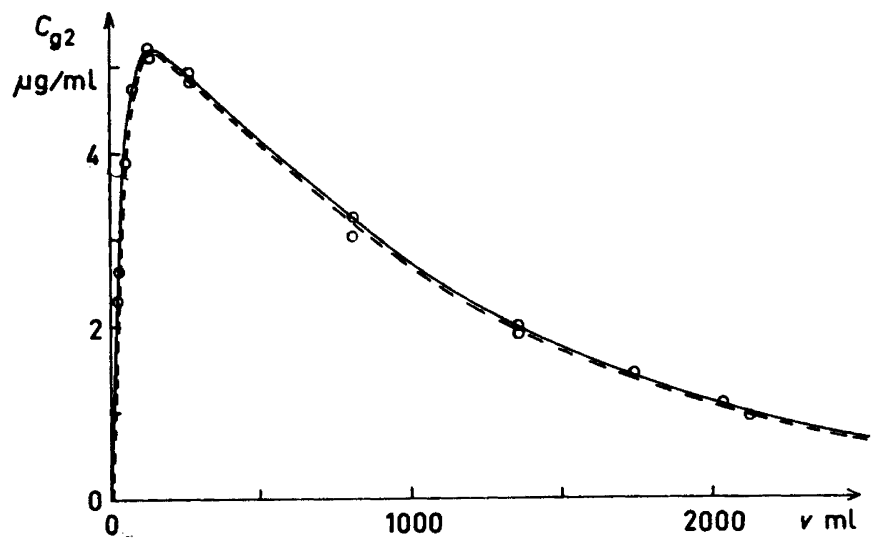

Fig. 3. Experimental test of eqn. 14. Solid line: theoretical curve, broken line: experimental curve. Parameters: $V_{\mathrm{L} 1} 102.5 \mathrm{ml}, V_{\mathrm{L}_{2}} 0.2 \mathrm{ml}, V_{\mathrm{g} 1} 67.5 \mathrm{ml}, V_{\mathrm{g}_{2}} 3.7 \mathrm{ml}, k_{1} 11$, $k_{2} 207, C_{\mathrm{g}_{0}} 5.97 \mathrm{~g} / \mathrm{ml}$. It is seen that $C_{\mathrm{L} 2 \max }$ is 16.5 times the zero time liquid concentration in the sample, and the yield $3 \%$. If the effective volumes had, for example, the values $V_{1}=10$ and $V_{2}=100$, the theoretical yield would be $77.5 \%$.

The volume of carrier gas, $v_{\max }$, corresponding to the maximum concentration in the collection tube, obtained by differentiation of (14), is

$$
v_{\max }=\frac{V_{1} \cdot V_{2} \cdot 2.3 \log \left(V_{1} / V_{2}\right)}{V_{1}-V_{2}}
$$

(15) becomes indefinite for $V_{1}=V_{2}$. In this case the correct value is obtained by interpolation.

Substituting (15) in (14), we obtain for the maximum amount which may be collected 


$$
\begin{gathered}
W_{2 \max }=C_{\mathrm{g} 2} V_{2}= \\
\frac{C_{\mathrm{g} 0} V_{1} V_{2}}{V_{1}-V_{2}}\left[\exp \left(-\frac{V_{2} \ln \left(V_{1} / V_{2}\right)}{V_{1}-V_{2}}\right)-\exp \left(-\frac{V_{1} \ln \left(V_{1} / V_{2}\right)}{V_{1}-V_{2}}\right)\right]
\end{gathered}
$$

From (16) it is seen that when the sample volume increases, other conditions being constant, the absolute amount of material collected increases. On the other hand, at higher $V_{1} / V_{2}$-values, the slope of the curve diminished and the carrier gas volumes required become uneconomically large. It is also seen that the smaller the effective volume of the sample is in relation to that of the collection vessel, the larger is the proportion of the eluted material collected which is to say (16) is a quantitative description of the fact that the distribution coefficients in the sample should be as small and those in the collection vessel as large as possible. The optimum carrier gas volume and the maximum amount of material which can be collected vary with the distribution coefficients of the compounds. Thus, if we wish to isolate some compounds preparatively by this method, the optimum conditions may be calculated from the distribution coefficients, which have to be determined, e.g. by making head-space chromatograms and using eqn. 2 in the form

$$
k_{\mathrm{i}}=\left(\frac{v}{\ln C_{\mathrm{g} 0}-\ln C_{\mathrm{g}}}-V_{\mathrm{g}}\right) / V_{\mathrm{L}}
$$

\section{EXPERIMENTAL}

It is important, in the quantitative determinations with concentration of the vapour, to make sure that a) the sample is adequately stirred, so that the carrier gas equilibrates with the sample, b) the carrier gas volumes passing through the sample are accurately measured, c) the vapours are efficiently trapped in the condenser, d) the temperature of the sample does not vary during elution, e) chemical changes do not occur in the sample during elution.

The test set-up for determinations with total condensation (eqn. 7) is shown in Fig. 4. Care was taken to ensure that the condensation path was long enough to allow the carrier gas to cool down adequately at the velocity used. When the volatiles had been collected from a volume of eluting gas, the condensation coil was connected in series with the column of a gas chromatograph, heated to $110^{\circ} \mathrm{C}$, and the carrier gas turned on. Some of the chromatograms were obtained with a Perkin-Elmer gas chromatograph $\mathrm{N}^{\circ}=800$, using a $1.8 \mathrm{~m}, 1.8 \mathrm{~mm} \emptyset$ butanediol succinate $(8 \%)$ column, temperature programme $30^{\circ}-130^{\circ}, 6.7^{\circ} / \mathrm{min}$, others with a P-E $116 \mathrm{E}$ model with a $2 \mathrm{~m}, 4 \mathrm{~mm} \emptyset$ polyethylene glycol column, $15 \%$ on PTFE, isothermally at $80^{\circ} \mathrm{C}$. HFI detectors were used.

The analytical method was tested with corn oil to which a mixture of pentane and 3 -heptanone had been added in amounts ranging between 250 and $500 \mu \mathrm{g} / 25 \mathrm{~g}$, and with homogenized apple to which a test mixture of acetaldehyde, propanol, 2-hexenal, and 2-

Fig. 4. Test set-up for quantitative determination with concentration of volatiles. 1, sample vessel, thermostat, magnetic stirrer. 2. Glass traps and coil of PTFE tubing (at least $1 \mathrm{~m}$ ), liquid nitrogen bath. (For suitable glass traps, see Ref. 5). 3, volumetric flask.

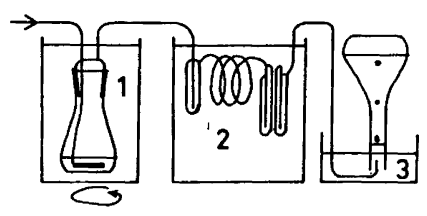

Acta Chem. Scand. 20 (1966) No. 1 

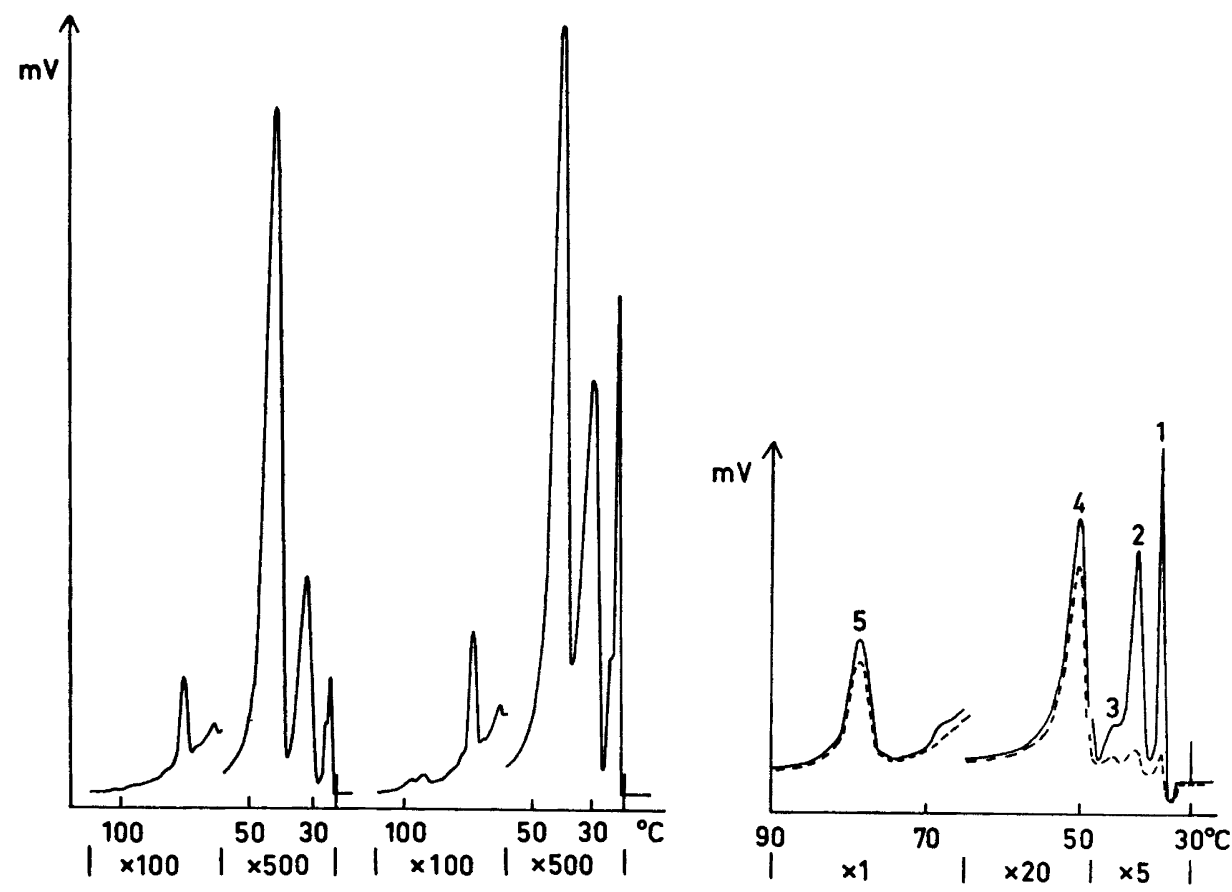

Fig. 5. The chromatograms from one quantitative determination of corn oil volatiles, where the volatiles were concentrated before chromatography. Butanediol succinate column, $1.8 \mathrm{~m}, 1.8 \mathrm{~mm} \emptyset$, gas flow rate $30 \mathrm{ml} / \mathrm{min}$, temperature programme.

Fig. 6. The chromatograms from a determination of bread volatiles by the headspace method with intermittent elution of the sample with inert gas. BDS-column. $1=$ pentane, 2 = furan, 3 and 4 unknowns, $5=$ 3-heptanone.

methyl-2-pentenal had been added, the amounts of the individual compounds ranging from 5 to $300 \mu \mathrm{g} / 100 \mathrm{~g}$. The apple was homogenized with about its own weight of water to render the homogenate easier to stir. Fig. 5 shows the two chromatograms from a determination of corn oil volatiles. The standard deviation for the determinations was $5.3 \%$. The average analytical recovery of the added compounds was $96 \%$. Because D.F. was 14 , this deviation from $100 \%$ was not significant.

A determination of volatiles from bread was made by the head-space method (eqn. 2, Fig. 6). $14 \mathrm{~g}$ of rye bread, ground with a homogenizer, was weighed into a stoppered conical flask of $300 \mathrm{ml}$ capacity. After the flask had stood for 10 min at room temperature with some stirring, $5 \mathrm{ml}$ of the gas phase was injected into the column with a gas-tight syringe, and the chromatogram run with temperature programming. Then $1000 \mathrm{ml}$ of nitrogen was led through the flask at a rate of about $25 \mathrm{ml} / \mathrm{min}$, while the sample was vigorously stirred, and a second $5 \mathrm{ml}$ of the gas phase was chromatographed. The result is shown in Table 1.

Table 1.

Compound added

Pentane

Furan

3-Heptanone
Concentration

$150 \mu \mathrm{g} / 100 \mathrm{~g}$

140

150
,

,
Analytical recovery

$$
\begin{aligned}
& 92.6 \% \\
& 94.5 \% \\
& 90.0 \%
\end{aligned}
$$


To test eqn. 14, nitrogen $(13.63 \mathrm{ml} / \mathrm{min})$ was led through a $170.0 \mathrm{ml}$ flask provided with a magnetic stirrer and containing a dilute solution of ethyl ether (7.1 $\mathrm{mg})$ in water $(102.5 \mathrm{ml})$, into a tube $(3.93 \mathrm{ml})$ containing $0.2 \mathrm{ml}$ of dodecane as solvent, and further to a flame ionization detector. The response curve, which gives $C_{\mathrm{g} 2}$ as a function of $v$, was recorded (Fig. 3). The temperature of the vessels was maintained at $27.5^{\circ} \mathrm{C}$. The distribution coefficients of ether between water and nitrogen $\left(k_{1}\right)$, and between dodecane and nitrogen $\left(k_{2}\right)$ were determined separately at $27.5^{\circ} \mathrm{C}$ by a method described earlier.

\section{DISCUSSION}

The methods described are not dependent on the chemical nature of the compounds to be determined (except perhaps for compounds which form ions in solution, for which the methods have not been tested), as long as we have some method to separate the eluted compounds from each other. A necessary condition is that the distribution coefficients do not change appreciably in the range within which their concentration changes during the determination. The methods were tested for compounds with boiling points from $21^{\circ}$ to $150^{\circ} \mathrm{C}$. For analysis of less volatile substances, liquid solvent extraction is probably preferable, because the carrier gas volumes to be used become excessive; in our hands a standard deviation of $5.3 \%$ was obtained. These methods may be applied for example to the analysis of food aromas, off-flavours, volatile food additives, or solvent residues. Because the method involves the determination of the distribution coefficients of the volatile compounds, it can be used to determine how much of a compound should be added to a material to achieve a certain vapour phase concentration above the material, or in deodorization work to calculate how large a gas volume should be used for flushing a material to reduce the concentration of some compound(s) to a predetermined level.

The equation describing the concentration curve in a condensation vessel containing solvent, when the condensation is incomplete, has its application when calculation of optimum conditions in preparative work is desired.

This work has been supported by a grant from Elintarvikkeiden Tutkimussäätiö (The Foundation for Food Research, Finland) which is gratefully acknowledged.

\section{REFERENCES}

1. Özeris, S. and Bassette, R. Anal. Chem. 35 (1963) 1091.

2. Weurman, C. Food Technol. 15 (1961) 531.

3. Mackay, D. A. M. Gas Chromatography, Scott, R. P. W. (Ed.), Butterworths, London 1960 , p. 357.

4. Wahlroos, Ö, Ann. Acad. Sci. Fennicae, Ser. A II 1963 No. 122.

5. Kimber, G. M. and Napier, D. H. Chem. Ind. (London) 1965725.

Received October 2, 1965. 